\title{
Design and Implement of Zigbee Network System Based on the Transpar- ent Transmission Mode
}

\author{
Zhengbing Zheng*
}

School of Physics and Telecommunication Engineering, Shaanxi University of Technology, Hanzhong, 723001, Shaanxi, China

\begin{abstract}
Currently main short-range communication technologies which are used to build wireless sensor network implementations conclude Bluetooth, WIFI technology and ZigBee technology. Zigbee technology has advantages in transmission distance, node capacity, battery life, etc. And ZigBee protocol formulates the network layer and application layer standard on the basis of IEEE 802.15.4 physical layer and data link layer standards, ZigBee technology is considered to be the best solution for wireless sensor networks. Wireless sensor network applications based on ZigBee technology mainly takes use of ZigBee protocol to implementnet working and data transmission. In order to simplify the implementation of ZigBee technology, this article put forward a kind of ZigBee Network system based on the transparent transmission mode. The system uses ZigBee firmware module to build the hardware platform, implements the control and functions of ZigBee firmware module through interface RS232 and implements the data transparent transmission inside network.Experimental tests show that: the network has a lower packet loss rate based on ZigBee network transparent transmission mode, reasonable packet length helps to reduce power consumption and improve the system stability, meanwhile extend the life cycle of the system.
\end{abstract}

Keywords: Wireless sensor networks, ZigBee technology, Zigbee protocol Stack, transparent transmission.

\section{INTRODUCTION}

Wireless sensor networks consist of a large number of low-power, tiny sensor nodes in the range of monitoring, is mainly used to achieve the target of monitoring and collecting the data of the monitoring object in the monitoring area .These sensor nodes are fixed or randomly deployed in the monitoring area and form a self-organizing system in a wireless communication way [1-3]. Between the sensor nodes there is a good collaboration, by means of various forms of highly integrated sensors, the sensor nodes can detect temperature, humidity, light intensity, water quality, and many other interesting physical objects. By connecting gateways, wireless sensor networks can access the Internet or existing mobile network and transfer the collected information to the terminal users. These features make the wireless sensor networks widely used in military, industrial control, environmental monitoring, smart home, precision agriculture and other areas.

Currently main short-range communication technologies which are used to build wireless sensor network implementations conclude Bluetooth, WIFI technology and ZigBee technology. Comparing from the number of transmission speed, transmission distance, battery life and network nodes, although the transmission rates of Bluetooth, WIFI technology are higher than that of ZigBee technology, but they are not as ZigBee technology in transmission distance, node capacity, battery life, etc [4-6]. ZigBee formulates the network layer and application layer standard on the basis of IEEE 802.15.4 physical layer and data link layer standards. ZigBee protocol achieved a function of self-organizing networks and selfmaintenance, which greatly facilitates the user to use, reduce network maintenance costs. Therefore, ZigBee technology is considered to be the best solution for wireless sensor networks.

Wireless sensor network applications based on ZigBee technology mainly takes use of ZigBee networking and data transmission protocol to implement networking and data transmission. To simplify the implementation of ZigBee technology, TI company has developed a specialized protocol stack software. ZigBee 2007 protocol stack software provides a stack scheduler called the operating system abstraction layer OSAL [7, 8]. For the development of users, except for being able to see the scheduler, any other specific implementation details of the protocol stack operations are encapsulated in the library code, the user does not know the specific details of the ZigBee protocol stack implemented only by calling the API interface complete a specific application development, can only complete a specific application development by calling API interfaces. Oherwise, the hardware platform TI's provide supports ZigBee 2007 protocol stack running on is mainly CC2530 chip that integrates an enhanced 8051 core, high performance $2.4 \mathrm{GHz}$ RF transceiver, capable of low-power wireless data transceiver [9]. Z-Stack is a ZigBee protocol stack software launched by 


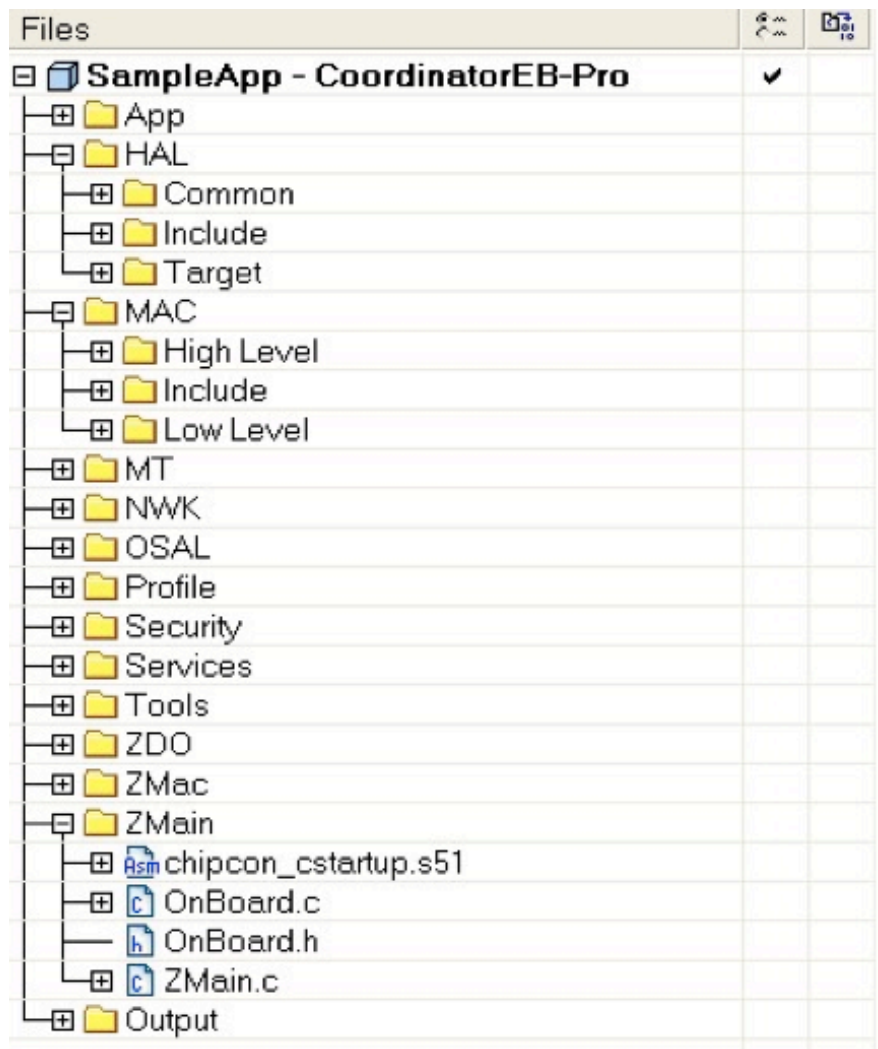

Fig. (1). ZigBee Protocol Stack Development Architecture.

TI's, providing a rapid realization of complete application templates for application developers ZigBee protocol stack development architecture is shown in Fig. (1).

Solutions based on ZigBee protocol stack software technology require a user familiar with API interface program, has some difficult to develop and extend the development cycle. In order to simplify the difficulty of ZigBee technology, this paper proposes a method based on the ZigBee network transparent transmission system that uses ZigBee firmware module. The system builds a hardware platform, control and function ZigBee firmware module through RS232 interface, the internal network will implements the transparent transmission of data.

\section{SYSTEM HARDWARE DESIGH}

\subsection{System Structure and Composition}

There are three main ZigBee network topology: star, tree, mesh-type [10].The topology of star network is simple, the information must be forwarded by the coordinator node between the terminal nodes, mainly used in the systems of smaller node number; Tree topology network covers a large range, if the distance between the nodes farther, need help forward the tree routing information to implement, and information transmission will be delayed too long; Mesh network topology communications cover a wide range of high reliability, multiple paths may exist between nodes, but requires more storage space. For wireless sensor networks can usually randomly be distributed sensor nodes in the monitoring area, and for the characteristic of building wireless network system in a self-organizing way, mainly study the design and implementation of ZigBee network system which is based on mesh network. Assumed that the wireless sensor network system architecture is as shown in Fig. (2).

The system mainly consists of the ZigBee network and the computer monitoring center. ZigBee network has a large number of monitoring nodes, the main types of nodes are : the coordinator node and router nodes and end nodes. Coordinator is the center of the entire network nodes, it will automatically select the frequency channel used by the network after being powered, network other nodes and make them join the network, provide routing information security management and data collection services. Router nodes are used to forward data between nodes and expand the network size. The terminal node is used to collect the data of the monitored object then transfer it to its parent. node, when not receiving data, it is usually dormant. ZigBee network node uses RS485 bus interface with the computer control center for data communication via the coordinator. Computer monitoring center is mainly composed by computer terminals and data servers. Usually use monitoring software to manage 


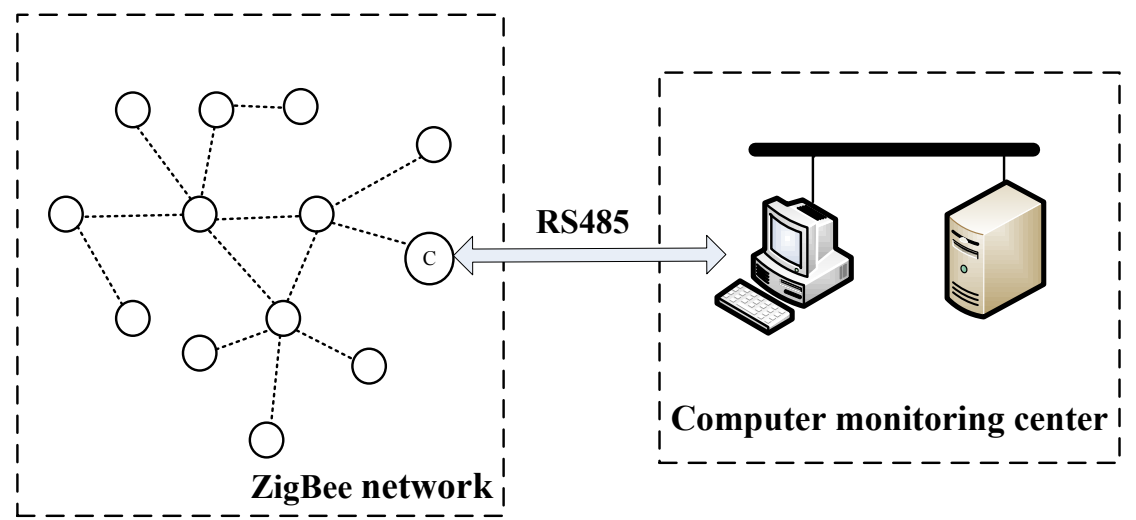

Fig. (2). The Wireless Sensor Network System Architecture

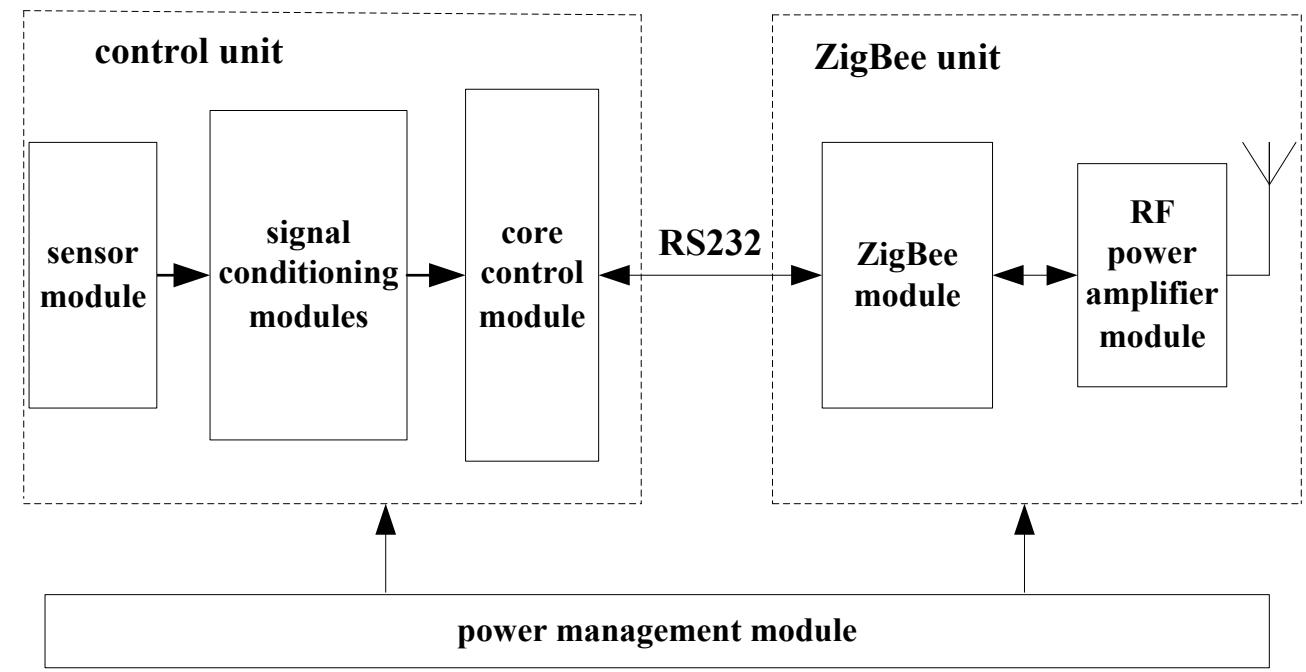

Fig. (3). Termination Node Hardware Structure.

access to external data, and achieve the node parameter setting, alarm monitoring, real-time graphical data display, data storage, data query.

\subsection{Node Hardware Design}

ZigBee network termination node hardware structure is shown in Fig. (3). Terminal node is made up of ZigBee unit and control unit. The control unit implements processing and the control on the signal of the external module and ZigBee unit implements the wireless data transmission between nodes. Control unit controls ZigBee unit controlled via RS232 bus.

ZigBee unit is a key part of achieving networking, it is mainly composed of ZigBee module and RF power amplifier modules. The implementation of ZigBee module completing protocol stack and the RF power amplifier module completing signal amplification are used to increase the signal transmission distance. The control unit consists of a sensor module, signal conditioning module and core control module. The sensor module is used to collect data information located in the terminal position of the object node, the signal conditioning module will appropriately amplify signal acquisition filter, and then sent to the core control module for data processing.

ZigBee network coordinator node and router node use the same hardware configuration, wherein the coordinator node hardware structure shown in Fig. (4). The terminal node is composed of ZigBee unit and core control unit. Core control module realizes signals processing and controlling to the external modules, ZigBee unit realizes the wireless data transmission between nodes. Core control module controls ZigBee control unit via RS232 bus.

The core control module of all nodes and ZigBee unit use the same hardware circuit. Core control module uses STM32F103ST's low-power processor STM32F103, based on the Cortex-M3 core chip architecture. The interior contains a 2-way 12-channel successive approximation type A DC module and 3 USA RT interfaces to meet the needs of 


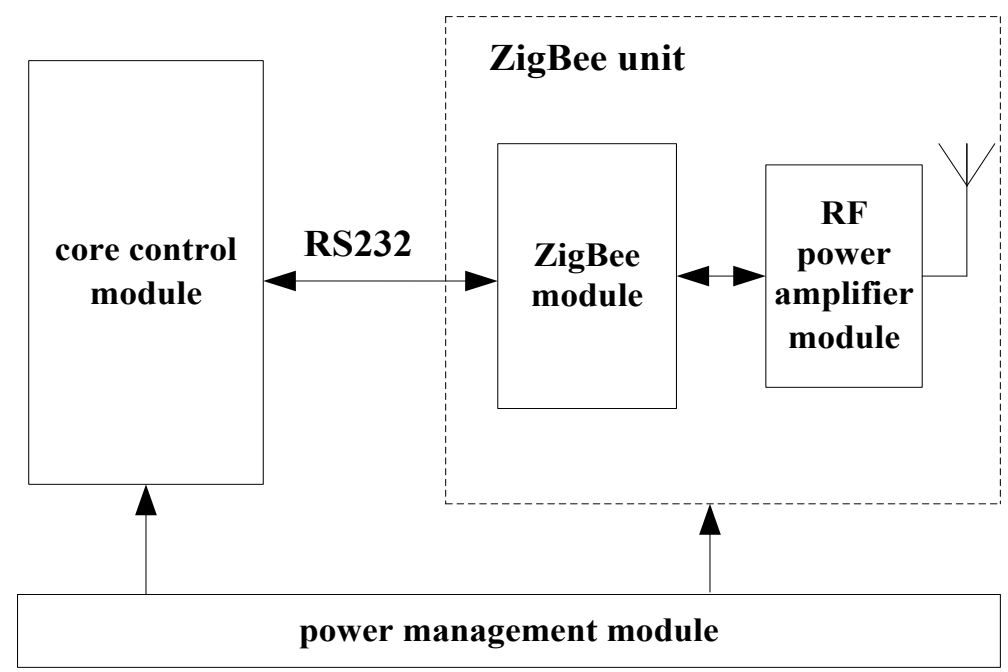

Fig. (4). Coordinator Node Hardware Structure.

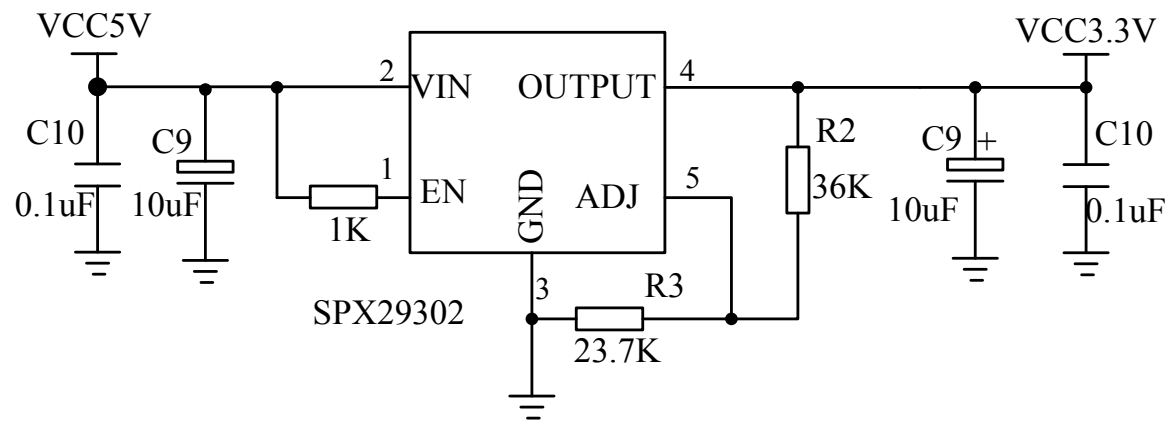

Fig. (5). A Circuit Diagram of the Power Management Module.

most applications. According to the power consumption and size requirements, ZigBee module uses TI's CC2530 as the main controlling chip, the chip is equipped with a special ZStack network protocol stack, integrated $2.4 \mathrm{GHz}$ RF transceiver of which the RF transmit output power is $4.5 \mathrm{dBm}$ and the receiving sensitivity is $-97 \mathrm{dBm}$; RF power amplifier module uses the highly integrated RF front-end, chip CC2591 from TI's. The interior of the chip integrated a power amplifier, a low noise amplifier, a balance converter, switches, inductors and a RF matching network. The maximum transmit power gain of $+22 \mathrm{dBm}$, receive gain up to 11 $\mathrm{dBm}$, which can significantly increase the system's wireless coverage [11].

That power management module provides a reliable power supply for the node is the key to ensuring the stable operation of the system. Node hardware is mainly powered by button battery. Button batteries provide $5 \mathrm{~V}$ voltage, the core control module and ZigBee module require $3.3 \mathrm{~V}$ voltage. Choose Sipex Semiconductor dropout linear regulator SPX2930as the 2 power management module, the chip provides a noise-free, owning a good stability of DC voltage, maximum output circuit reaches $3 \mathrm{~A}$, possessing as excellent transient response at the same time. A circuit diagram of the power management module is shown in Fig. (5). By dividing resistors $\mathrm{R} 2$ and $\mathrm{R} 3$, the conversion from $5 \mathrm{~V}$ to $3.3 \mathrm{~V}$ voltage gets realized.

\section{SYSTEM NETWORK DESIGN}

To simplify the design requirements, ZigBee modules adopt the Tektronix Electronics Co., Ltd. Shenzhen Ding DRF1605H-CC2530 module, of which the main chip is based on the C2530F256 chip that can run ZigBee2007 agreement directly. ZigBee module automatically complete all of the ZigBee protocol processing section, users do not need to understand the complex ZigBee protocol, you can implement all network functions to transfer data, only through the serial port

\subsection{Module Parameter Configuration}

DRF1605H-CC2530 module can be used as soon as is powered, users can complete the configuration parameters through specialized instruction, these parameters are configured after power-down save. ZigBee module of configurable parameters command is as shown in Table 1. Serial data rate 
Table 1. ZigBee Module's Primary Configurable Parameter Order.

\begin{tabular}{|c|c|c|}
\hline Function & Order & Directive Parameter Specification \\
\hline Serial port parameter & FC 019106 XX F6 XY & $\begin{array}{c}\text { Set XX } \\
\text { Confirm series rate }\end{array}$ \\
\hline The wireless channel parameter & FC $01910 C$ XX 1A XY & $\begin{array}{c}\text { Set XX } \\
\text { Confirm the module of channel }\end{array}$ \\
\hline PANID number & FC 029101 XXXX XY & $\begin{array}{c}\text { Set XXXX } \\
\text { Confirm network signal } \\
\text { No connection between different PANID。 }\end{array}$ \\
\hline \multirow{2}{*}{ Types of node } & FC 009109 A9 C9 XY & Set module as Coordinator \\
\hline & FC 00910 A BA DA XY & Set module as Router \\
\hline Work model & FC 01916458 XX XY & $\begin{array}{c}\text { Set XX } \\
\text { Confirm data transparent transmission mode }\end{array}$ \\
\hline
\end{tabular}

Table 2. The Form of Data Transmission Order.

\begin{tabular}{|c|c|c|c|c|c|}
\hline $\begin{array}{c}\text { Send } \\
\text { Form }\end{array}$ & $\begin{array}{c}\text { Point-to-Point } \\
\text { Binding ID }\end{array}$ & $\begin{array}{c}\text { Length of } \\
\text { Data }\end{array}$ & $\begin{array}{c}\text { Target } \\
\text { Address }\end{array}$ & Data (Maximum=32 Bytes) \\
\hline \hline Example & FD & 06 & $140 \mathrm{E}$ & 010203040506 \\
\hline $\begin{array}{c}\text { Receive } \\
\text { Form }\end{array}$ & $\begin{array}{c}\text { Point-to-point } \\
\text { Binding ID }\end{array}$ & $\begin{array}{c}\text { Length Of } \\
\text { Data }\end{array}$ & $\begin{array}{c}\text { Target } \\
\text { Address }\end{array}$ & Data (maximum=32 bytes) \\
\hline Example & FD & 06 & $140 \mathrm{E}$ & 010203040506 \\
\hline
\end{tabular}

is used to determine the parameters of command between the external processor and ZigBee module serial transmission, usually baud rate is set to 38400 , at this moment the maximum packet can be transmitted at a time is 256 bytes; radio channel parameters command is used to determine the node or the entire network operating frequency; PANID Directive to determine the ZigBee network number; node type instruction determination module is the coordinator node or router node; mode command to determine the module's data transmission. There are eight specific data transmission are: transparent data transmission mode, transparent transmission + short address mode, transparent transmission + MAC mode, transparent transmission + custom address mode, point to point data transmission + ZigBee short address addressing mode, point to point data transmission + to Baotou trailer + ZigBee short address addressing mode, point to point data transmission to the header + trailer + ZigBee short address addressing mode, point to point data transmission to the header + trailer + custom address addressing, reliable transmission mode.

\subsection{Node-to-node Data Communication Process Design}

ZigBee network coordinator node is used to create a Zigbee network, each Zigbee network has one and only one
Coordinator. When a node is added, the distribution network short address to the child nodes, and will always be in working condition. Router node is responsible for finding the most suitable route between nodes path to forward packets, when a new node is added, a short address can be assigned to the node network. Each Zigbee network is automatically routed to the destination node.

Since DRF1605H-CC2530 module can be only set to coordinator node type and node type router, in order to achieve mutual communication between the coordinator node and the terminal node, there must be logical operation between the nodes, this case chooses point-to-point transferring + ZigBee short address addressing mode and transparent data transmission.

\subsubsection{Point-to-point Data Transmission + ZigBee Short Address Addressing Mode}

After the ZigBee network is successfully set up, each of the network router nodes are assigned a unique short address, when data are transferred between the nodes, the destination and source addresses can be determined through the network. The mode data instruction format is shown in Table 2. Can be seen from Table $\mathbf{2}$, the received data format adds source address information to the tail of the packet based on a 


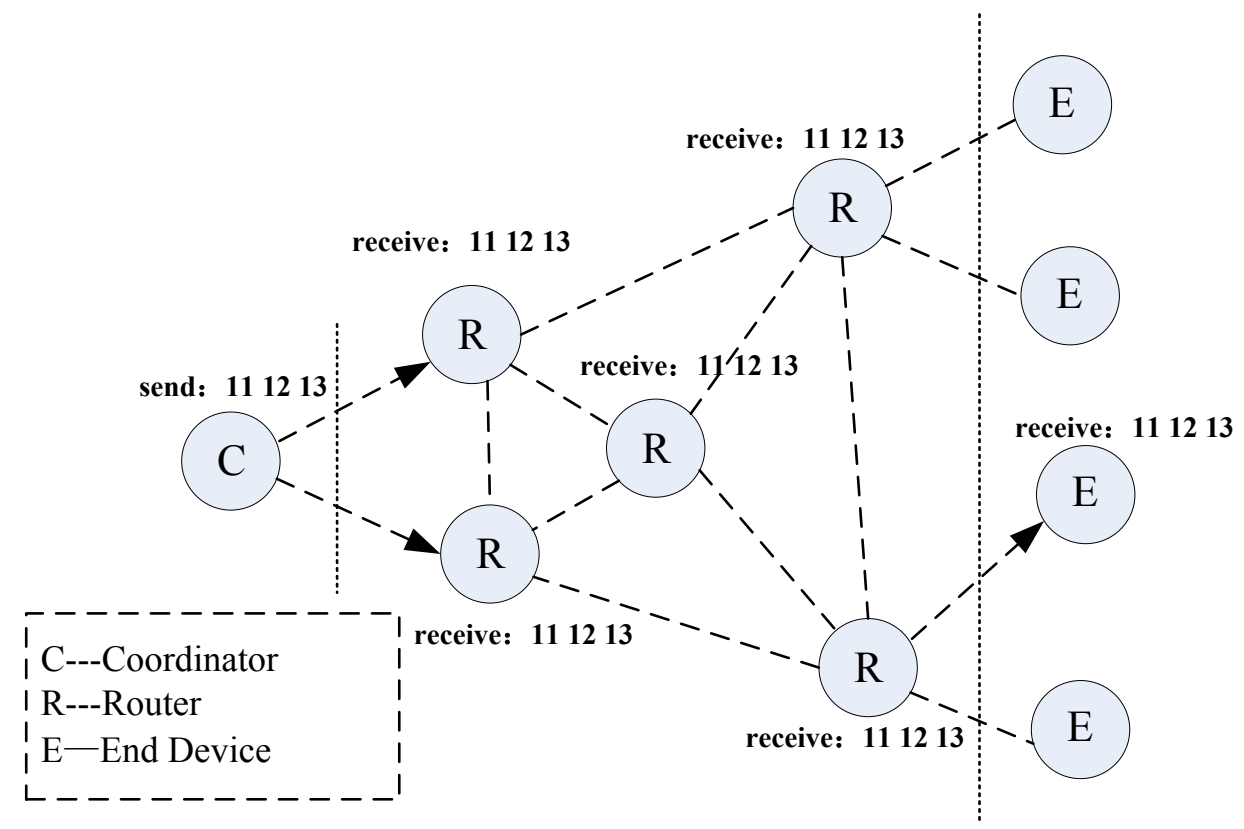

Fig. (6). The Process that Data Transmits From A Coordinator Node to A Terminal Node.

transmission data format, the receiving node will determine the location of the sending node based on the source address information. The length of data-sending can be modified, but it cannot be more than 32 bytes, the packet header identifies the data transfer mode FD.

\subsubsection{Transparent Data Transmission}

According to data transmission flag signal, as long as the first byte transmitted is not $0 \mathrm{xFE}, 0 \mathrm{xFD}$ or $0 \mathrm{xFC}$, the node will automatically enter the transparent data transmission. The data the coordinator node receives from the serial port will automatically be sent to all nodes; The data a node receives from the serial port will be automatically sent to the coordinator node. There is no need to set, and it supports packet variable, to a maximum of 256 bytes per packet.

\subsubsection{The Coordinator Node to Transmit Data to The Ter- minal Node Process}

In order to reduce the overall power consumption of the network system, assuming the relative position of the fixed nodes, and the terminal nodes bind with the corresponding node router, i.e., the router node terminal node binding (generally 1-5) are managed. Fig. (6) shows the process that data transmits from a coordinator node to a terminal node. Assuming send data to 111,213 , the coordinator node sends data to the router node in a transparent data transmission mode, the nearest the location to the node of all the routers send data were received for 111213 , of which the terminal node router nodes bound to point data sends data to the terminal node in the way of addressing transferring + ZigBee short address. Router nodes throughout the network makes up transmission network to provide suitable data path for the coordinator node to the terminal node to transmit data.

\subsubsection{Terminal Nodes Transmit Data to The Coordinator Node Process}

The process of terminal node transmitting data to the coordinator node is shown in Fig. (7). Assuming the data transmitted to terminal node are 111213 , the terminal node sends data to the router node terminal node in the way of point-to-point data transmission + ZigBee short address addressing mode, the router node uses the network to select the appropriate transmission path to transmit data the to the coordinator node in a transparent data transmission mode.

\subsubsection{The Terminal Node and Router Nodes Binding Im- plementation}

Between the terminal node and router nodes there exist the point to point data transmission + ZigBee short address addressing communication, so that redefining the contents of the transmission data format helps to complete the binding. Specifically: choose the first byte of data as a management identifies a terminal node, the other is the corresponding data byte. Thus, the router node conduct a numbering management to terminal node, using the number information to transmit data to the corresponding terminal node .

\subsection{The Implementation of Networking Processing}

The network networking process is: First, configure the software settings for a module node type as Coordinator (the coordinator node), and then restart the module, set the PAN ID coordinator node (range: 0x0001 -0xFF00), after setting 0xFF00) turn off power; then, set the PAN ID of the router (router nodes) that need to join this network to 0xFFFF, automatically find any network, after setting turn off power; and finally, charge the Coordinator, then charge other routers, it can automatically join the network, after a few 


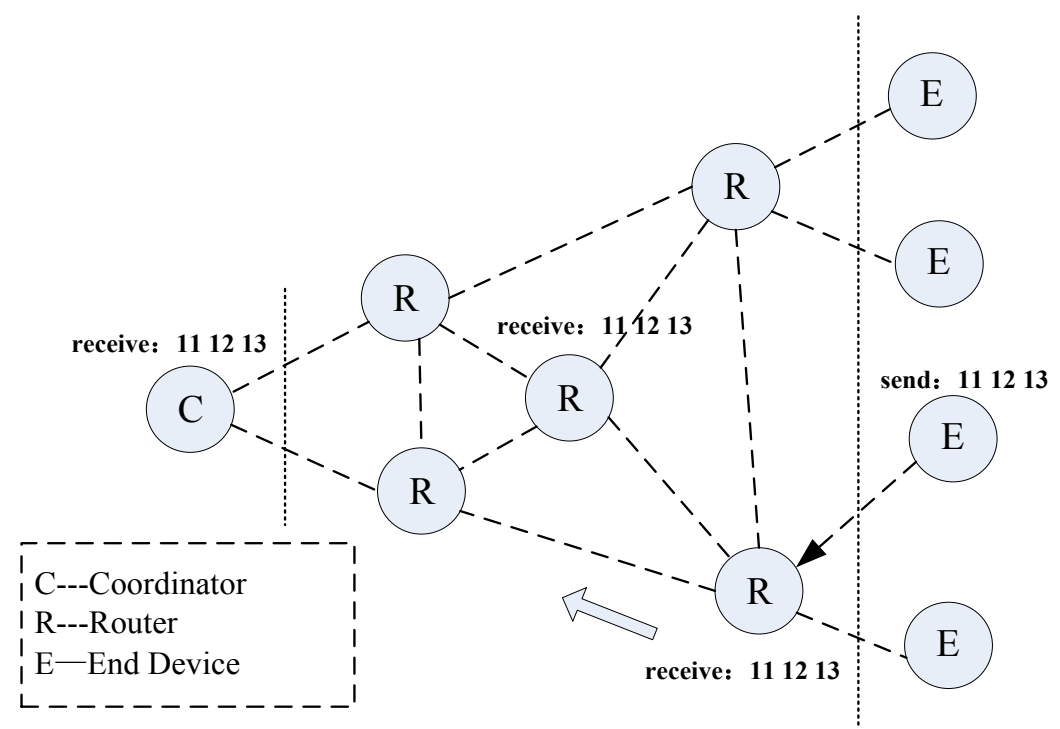

Fig. (7). The Process of Terminal Node Transmitting Data to The Coordinator Node.

Table 3. The Statistical Results of Tests of Transmission Packet Loss Rate.

\begin{tabular}{|c|c|c|c|}
\hline Node Number & Send Packet $\mathbf{~}$ & Receive Packet M & Transmission Packet Loss Rate Y\% \\
\hline \hline 01 & 1000 & 996 & 0.4 \\
\hline 02 & 1000 & 1000 & 0.0 \\
\hline 03 & 1000 & 990 & 1.0 \\
\hline 04 & 1000 & 985 & 0.7 \\
\hline 05 & 1000 & 993 & 0.5 \\
\hline 06 & 1000 & 995 & 0.5 \\
\hline
\end{tabular}

seconds, the network set up is completed. In the process of the network running, it will complete the data transfer between the different terminal nodes and the coordinator node.

\section{SYSTEM EXPERIMENTS AND RESULTS ANALYSIS}

\subsection{Tests of Transmission Packet Loss Rate}

Set each terminal node sends data to the coordinator node every 10 minutes, once 1000 packets, denoted as $\mathrm{N}, \mathrm{t}$ he node sends a packet into receive mode, waiting for forwarding packets every other node. The number of packets received coordinator node, denoted as $\mathrm{M}$, and the test terminal node to the coordinator node ransmits the packet loss rate, denoted as Y. The statistical results is shown in Table 3 . Wherein the loss rate is calculated as:

$\mathrm{Y}=\frac{M-N}{N} \times 100 \%$
The average loss rate of $0.68 \%$ of the entire network. Be seen, the system has the characteristics of low packet loss rate and high reliability.

\subsection{Propagation Delay Test}

In the process of data transmission, the size of the data frame directly affects the data transmission delay. According to the data transfer instruction format, when the node is operating in transparent data transmission mode, the shortest delay time testing coordinator node sends data to the router node relationship with inter-node data packet length is shown in Fig. (8). Test condition asks $10 \mathrm{~m}$ distance between nodes, continuous transmission of $100 \mathrm{~K}$ bytes, testing error-free, then test 10 times continuously.

As can be seen in Fig. (8), under the condition of uncensored transmission, the node distance is fixed, the larger the data packets between nodes, the longer the time it takes to transfer, along with the increasing number of network nodes, the amount of the information interaction between nodes in this case will increase greatly, the delay between the nodes 


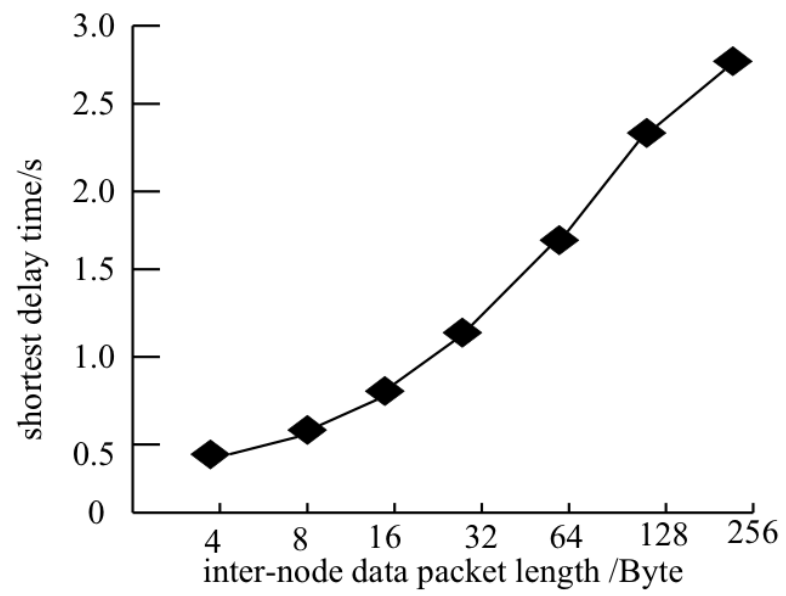

Fig. (8). The Shortest Delay Time Testing Coordinator Node Sends Data to The Router Node Relationship with Inter-node Data Packet Length.

will increase and the overall power consumption of the system will increase, the life cycle of the system will be greatly reduced, and therefore, reasonable data packet length stabilization system helps to reduce power consumption and improve system, it is recommended to set the data packet length 32 bytes.

\section{CONCLUSION}

The system uses ZigBee firmware module to design ZigBee network node, with the benefits of simple hardware structure, versatility, flexible extension etc. ZigBee firmware module solidified ZigBee protocol stack, under the control of The external processor, it can work in different operating modes, completing the transparent transmisson of ZigBee network data through simple parameter configuration. After the system transmission packet loss and transmission delay node tests, ZigBee network based on the transparent transmission system bears a better reliability and stability, when the packet length is smaller, the life cycle of the system will be extended.

\section{CONFLICT OF INTEREST}

The authors confirm that this article content has no conflicts of interest.

\section{ACKNOWLEDGEMENTS}

The work was supported by the project of National Natural Science Fund (No. 61401262) and Scientific Research Program Funded by Shanxi Provincial Education Department (No. 2013JK1059).

\section{REFERENCES}

[1] Y. Pan, and L. Sun, "Design and implementation of the greenhouse environment monitoring system based on zigbee wireless network" Computer and applied chemistry, vol. 31, no. 7, pp. 807-810, 2014.

[2] Z. Zheng, "Design of a wireless monitoring System for marsh gas project based on nRF24L01 and GSM" Nuclear Electronics \&and Detection Technology, vol. 33, no. 8, pp. 995-999, 2013.

[3] Y. Cai, G. Liu, L. Li, H. Liu, "Design and test of nodes for farmland data acquisition based on wireless sensor network", Transactions of the Chinese Society of Agricultural Engineering (Transactions of the CSAE), vol. 25, no. 4, pp. 176-178, 2009.

[4] X. Peng, T. Zhang, Y. Cheng, J. Li, "Application of wireless sensor networks in the field of agriculture" Journal of Agricultural Mechanization Research, no. 8, pp. 245-248, 2011.

[5] . Zhang, X. Yu, and G. Bi, "Research and realization of wireless sensor networks" Journal of CUMT Mining Science and Technology, vol. 36, no. 5, pp.128-134, 2007.

[6] W. Zhang, X. Yu, and Z. Li, "Wireless network sensor node design based on CC2530 and ZigBee protocol stack", Computer Systems and Applications, vol. 20, no. 7, pp. 184-187, 2011.

[7] R. Zhang, G. Gu, Y. Feng, Z. Huang, "Realization of communication in wireless monitoring System in greenhouse based on IEEE", Transactions of the Chinese Society for Agricultural Machinery, vol. 39. no. 8, pp. 119-122, 2008.

[8] J. Han, C.S. Choi, I. Lee, "More efficient home energy management system based on ZigBee communication and infrared remote controls," IEEE Trans Consum Electron, vol. 57, no.1, pp. 85-89, 2011.

[9] G. Vellidis, M. Tucker, C. Perry, C. Kvien, "A real-time wireless smart sensor array for scheduling irrigation" Computer and Electronics in Agriculture, vol. 61, no. 1 pp. 44-50, 2008.

[10] F. Ye, G. Zhong, S.W. Lu, L.X. Zhang, C. Kvien, "Gradient broadcast: A robust data delivery protocol for large scale sensor networks" ACM Wireless Networks, vol. 11, no. 3, pp. 285-298, 2008.

[11] L. Ruiz-Garcia, P. Barreiro, and J. I. Robla, "Performance of Zigbee-based wireless sensor nodes for real-time monitoring of fruit logistics," Journal of Food Engineering, vol. 87, pp. 405-415, 2008. 